\title{
CORRELATION BETWEEN SIMULATIONS AND REAL MEASUREMENTS OF MICROSTRIP FILTERS BASED ON LTCC IN HIGH FREQUENCY AREA
}

\author{
Kornel RUMAN, Tibor ROVENSKÝ, Alena PIETRIKOVÁ \\ Department of Technologies in Electronics, Faculty of Electrical Engineering and Informatics, \\ Technical University of Košice, Letná 9, 04200 Košice, Slovak Republic, tel. +421 55602 4220, e-mail: kornel.ruman@tuke.sk
}

\begin{abstract}
The aim of this paper lies in comparison of two CAD (Computer aided Design) software (Ansoft Designer from Ansys and HyperLynx 3D EM Designer from Mentor Graphics) and real measurements of designed and fabricated filters. Both software tools belong to the group of the most used tools for design and simulation of different structures. These structures are made by transmission lines of a few kinds (microstrip, stripline, slotline, etc.) and they substitute common elements in HF (High Frequency) area. This paper presents 3 various microstrip filters based on LTCC (Low Temperature Co-fired Ceramic) dielectric material GreenTape 951. Low pass (LP), band pass (BP) and band stop (BS) filters for UWB (Ultra Wide-Band) sensor systems were designed, simulated, fabricated and measured. Measured results and simulations (S21 - forward transmission coefficient) are plotted in joint graphs where divergences are observed and depicted.
\end{abstract}

Keywords: CAD, Filters, LTCC, scattering parameters

\section{INTRODUCTION}

Nowadays, there are a variety of CAD programs for EM (Electromagnetic) simulation and design of any HF area planar structures on the market. Choosing the best software tool is very difficult because each one has many advantages and disadvantages, therefore the choice depends mainly on the designers and their needs. For the purposes of filters design it is important to select a software tool which provides many options relating to substrate parameters and simulations. When the filters are designed for HF area, there is necessity for $\mathrm{S}$ parameters (Scattering parameters) simulations.

With rising requirements for minimalizing dimensions with combination of more complex electronic devices CAD software tools started to be very important part of filter design. Nowadays, they are very sophisticated tools which imply not only drawing of schemas and their storage in electronic form, but they also provide many options how to perform simulations, real - time tuning of designed structures, selecting or even specify any used substrate and conductive material. Besides the mentioned options CAD software tools can generate files for fabrication designed devices e.g. Gerber files etc.

Each CAD software tool has its own algorithm for counting results and simulations done by different tools that could provide different results. It is very important to verify correlation between simulations and real measured results for each tool. Based on these comparisons designers can pick out the most suitable CAD software tool for their purposes.

Advances in CAD, such as full-wave EM simulators, did coup in design of microstrip filters. In this paper, for the design of the three different microstrip filters we used software Ansoft Designer and HyperLynx 3D EM Designer, which facilitated the design of microstrip filter based on LTCC.

\section{SOFTWARE TOOLS}

From the large variety of the CAD software tools on the market, for the structures' design Ansoft Designer from Ansys and HyperLynx 3D EM Designer from Mentor Graphics were chosen. Both of these CAD software tools offer a few types of simulations, including the most required and used EM simulation which is an advanced technology to yield high accuracy analysis and design of complicated microwave and RF (Radio Frequency) printed circuit, antennas, high-speed digital circuits and other electronic components.

\subsection{Ansoft Designer}

Ansoft Designer from Ansys has two types of simulations: LNA (linear network analysis) and full EM. LNA is based on simple mathematical model and give ideal results because it does not take into account advanced parameters which could influence simulations. LNA is not computationally difficult and takes very short time, therefore real - time tuning is very easy to perform. EM takes into account more parameters, simulation is more complex, and time to get simulations' results is incomparably longer than LNA [1].

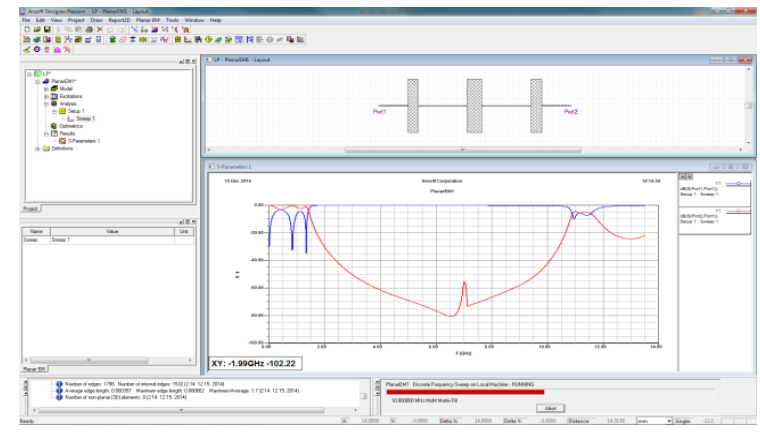

Fig. 1 Ansoft designer screen [1] 


\subsection{HyperLynx 3D EM Designer}

HyperLynx 3D EM Designer IE3D is an integrated fullwave EM simulation and optimization package for the analysis and design of 3D and planar microwave circuits, antennas, digital circuits and high-speed PCB (Printed Circuit Boards). Since its formal introduction in 1993 IEEE International Microwave Symposium, the IE3D has been adopted as an industrial standard in planar and 3D electromagnetic simulation. The IE3D has become the most versatile, easy to use, and efficient EM simulation tool [2].

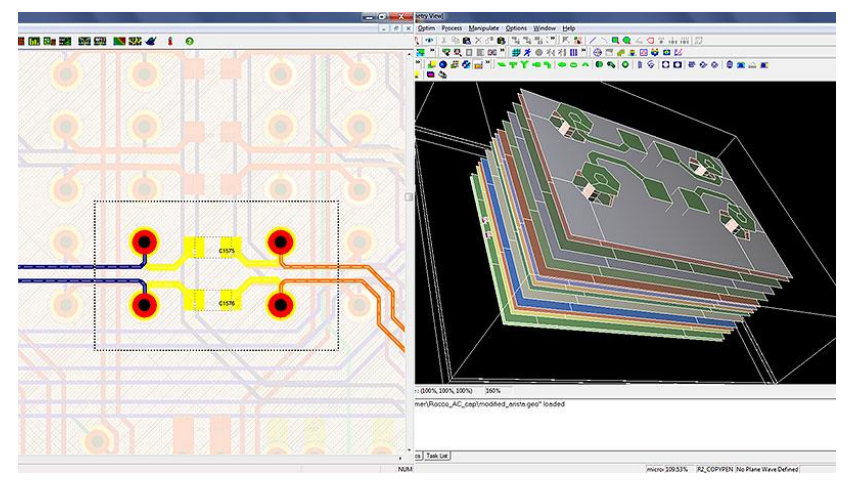

Fig. 2 HyperLynx 3D EM Designer screen [2]

\subsection{Advantages and Disadvantages}

Advantages and disadvantages mentioned here are concerning only HF microstrip structure design which is the main topic of our research area.

Both software tools have their own way of how to design microstrip circuits which is necessary to be understood before designing certain structure (e.g. filter, power divider, antenna, etc.). They have no limitation for number of dielectric layers which does not limit design of 3D structures based on LTCC.

Ansoft's biggest advantage is that the simulation also contains the information for real-time tuning where one can observe changes of simulations at the same time as the dimensions of structures are changed.

Mentor's has advantages in specifying parameters of conductive materials which influenced performance of structures and the most valuable benefit lies in specifying properties of the used dielectric substrates including option to set these properties according to exact frequencies. The EM simulation time is shorter in comparison with Ansoft, but does not include information for real-time tuning kit. Mentor provide real-time tuning of designed structures but it is necessary to define correct variables and re-run the simulation.

\section{FILTER DESIGN}

\subsection{Dielectric Material}

All filters are fabricated by a thick film technology using LTCC substrate DuPont GreenTape 951PX which belongs to GCC (Glass Ceramic Composites). Typical glass content of this GCC varies between $20 \%$ and $50 \%$ [3]. Microstrip structure is made by silver conductive paste with high electrical conductivity and typical sintering temperature below $900{ }^{\circ} \mathrm{C}$. For LTCC dielectrics, there is a need for a low thermal expansion, lower thermal resistance, and high mechanical strength as well. Green Tape 951 is available in multiple thicknesses and it is designed to be used as an insulating layer in multichip modules, single chip packages, ceramic printed wiring boards and RF modules.

Before designing any structure it is necessary to know physical and electrical parameters of the used substrate. Dielectric constant of DuPont GreenTape 951 is 7.8 and loss tangent is 0.006 [4].

Low Dissipation Factor Tan $(\delta)$ allows this LTCC material system to be used in many applications where at higher operating frequency the conventional laminates (FR4) circuit boards are limited. In addition its higher dielectric constant in comparison with other dielectric materials (FR4, Rogers RO4003C) allows designing smaller microstrip structure what leads to miniaturization of the whole designed system.

\subsection{Dielectric material}

Conductive paste was chosen based on the value of the fired resistivity because this parameter influences the transmitted signals' quality (the smaller the better). LTCC ceramics does not have zero shrinkage after firing process in axis $\mathrm{x}, \mathrm{y}$ and $\mathrm{z}$, and for this reason the structures of the microstrip BP, BS and LP filters were printed with post fire paste LF171 AgPt. The resistivity of this post fired paste is less than $2.5 \mathrm{~m} \Omega / \mathrm{sq}$. Post fire printing process eliminates the differences in dimension between designed and fabricated microstrip filters [5].

\subsection{Low Pass Filter}

Requirements on microstrip LP filter are cut off frequency $2.5 \mathrm{GHz}$ and minimum attenuation $-40 \mathrm{~dB}$ in stop band to provide antialiasing of processed signal in UWB sensor system [6]. Based on the comparison result of various possibilities for the shape of microstrip LP filter that offer CAD software, we decided for steppedimpedance type of structure. The Fig. 3 shows the structure of five-pole stepped-impedance microstrip LP filter (with its dimensions), which used a cascaded configuration of alternating high and low impedance transmission lines [7, $8]$.

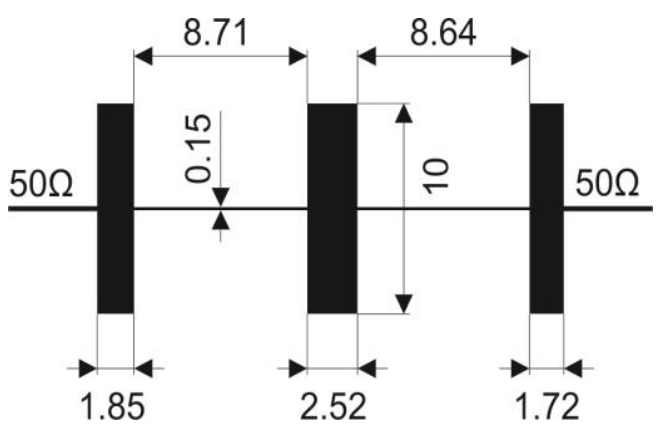

Fig. 3 A layout of the designed microstrip LP filter for LTCC substrate GreenTape 951PX (unit: $\mathrm{mm}$ )

\subsection{Band Pass Filter}

The designed BP filter should be used in I - Q demodulator presented in [9] which is a part of laboratory UWB sensor system. UWB sensor systems must match the 
ECC (Electronic Communications Committee) frequency bandpass and therefore requirements on BP filter include operating bandwidth from 6 to $8 \mathrm{GHz}$ and a minimum attenuation $-40 \mathrm{~dB}$ in stop band [10].

Based on the comparison results of various possibilities for the shape of microstrip BP filter (Microstrip GapCoupled Bandpass filter, Microstrip Parallel-Coupled Bandpass filter, Microstrip Hairpin Bandpass filter, etc.) that offer CAD software, we decided for hairpin type of structure. The Fig. 4 shows six-pole microstrip BP filter, which is designed as a cascade of parallel resonant circuits, among which is also capacitive coupling. Resonant circuits are realized using half wave U-resonators (also called hairpin resonators) and capacitive coupling through their mutual distance $[7,11]$.

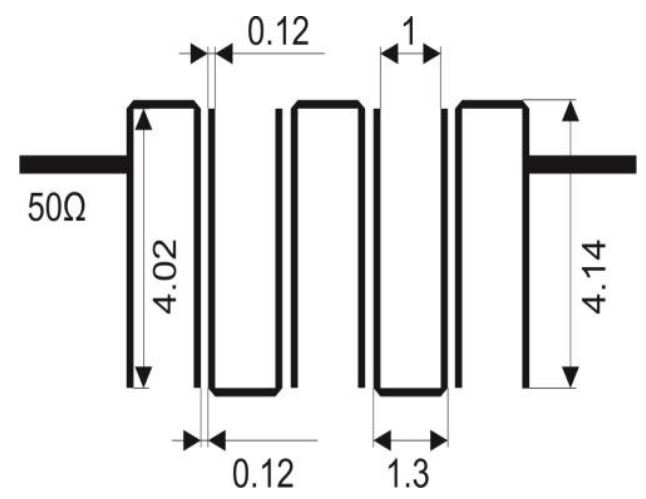

Fig. 4 A layout of the designed microstrip BP filter for LTCC substrate GreenTape 951PX (unit: mm)

\subsection{Band Stop Filter}

Narrowband BS filter is shown in Fig. 5 and it was designed to minimalize interference and degradation of useful signal for UWB sensor system caused by Wi-Fi signal [12]. Microstrip BS filter consists of three half wavelength L-resonators and feed line. Resonators are bended to reduce dimensions of designed filter. Stopband of this filter is from $2.4 \mathrm{GHz}$ to $2.5 \mathrm{GHz}$ with required attenuation at least $-40 \mathrm{~dB}$. Filter has pads for connectors which matched characteristic impedance $50 \Omega$ [13].

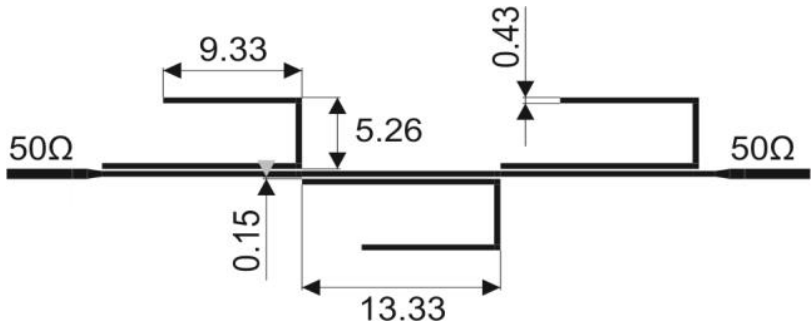

Fig. 5 A layout of the designed microstrip BS filter for LTCC substrate GreenTape 951PX (unit: $\mathrm{mm}$ )

\section{SIMULATED AND MEASURED RESULTS}

Simulated and measured results are plotted in one joint graph. Blue curve represents the measured results provided by Vector Network Analyser from $0 \mathrm{GHz}$ to $13.5 \mathrm{GHz}$. SMA female connectors were soldered to each filter and connected with VNA using test port cables with SMA male connectors. VNA was calibrated before measurements of each filters' type to provide better accuracy. Red curve is simulated forward transmission parameter by HyperLynx 3D EM Designer and green curve represents simulated forward transmission parameter by Ansoft Designer.

In Fig. 6 frequency domain characteristic of microstrip LP filter is shown. Simulations and measured results have a lot of divergences and different attenuation in stopband. From $0 \mathrm{GHz}$ to $4 \mathrm{GHz}$ simulations provided by HyperLynx 3D EM Designer matched with measured results. Above the frequency $4 \mathrm{GHz}$ the transmission characteristics of measured LP filters does not achieve attenuation as simulations but it is still acceptable.

Fig. 7 shows frequency domain characteristic of BP filter. Simulation provided by HyperLynx 3D EM is in very precise match with measured results and simulations by Ansoft Designer correlated with measured curve with small divergences mostly in pass band.

In Fig. 8 frequency domain characteristic of BS filter is shown. Simulation made by Ansoft Designer shows greater attenuation in stopbands than real measurements really prove, on the other hand, these measured results are more correlated with simulations by HyperLynx 3D EM.

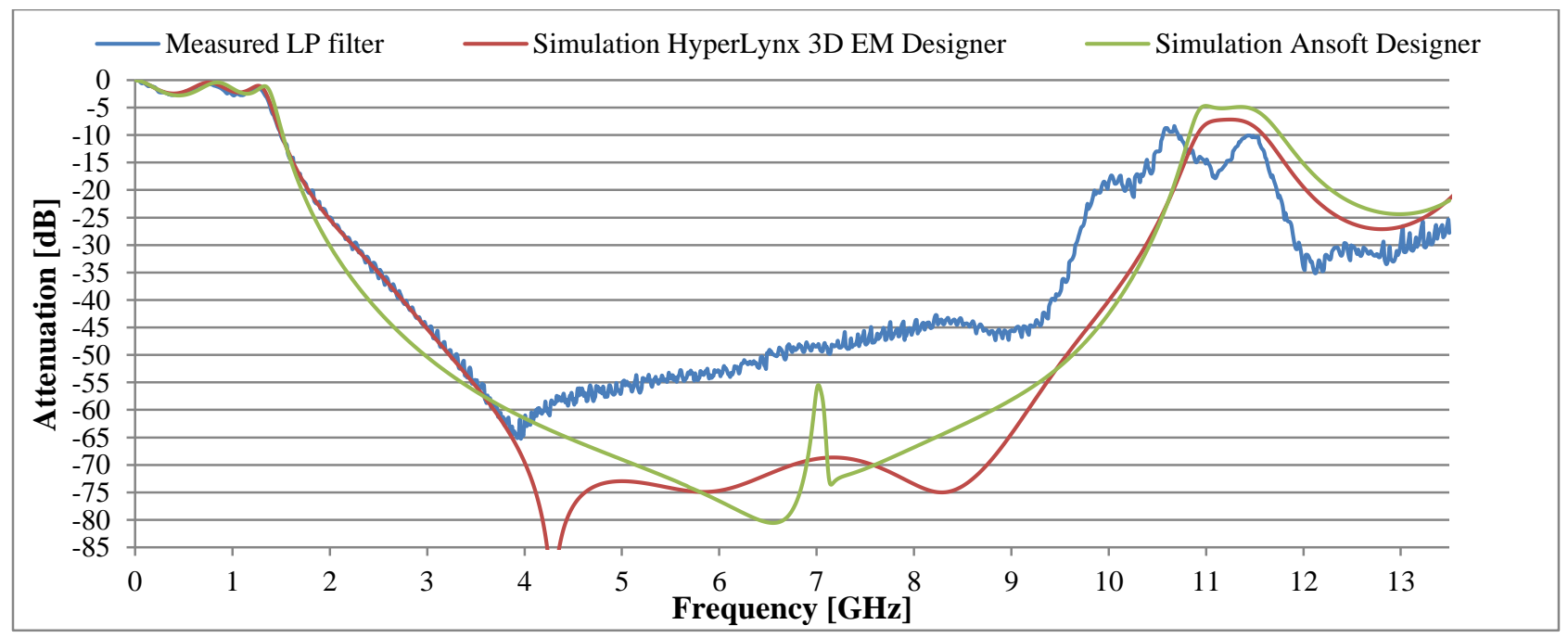

Fig. 6 Measured and simulated forward transmission coefficient of LP filter 


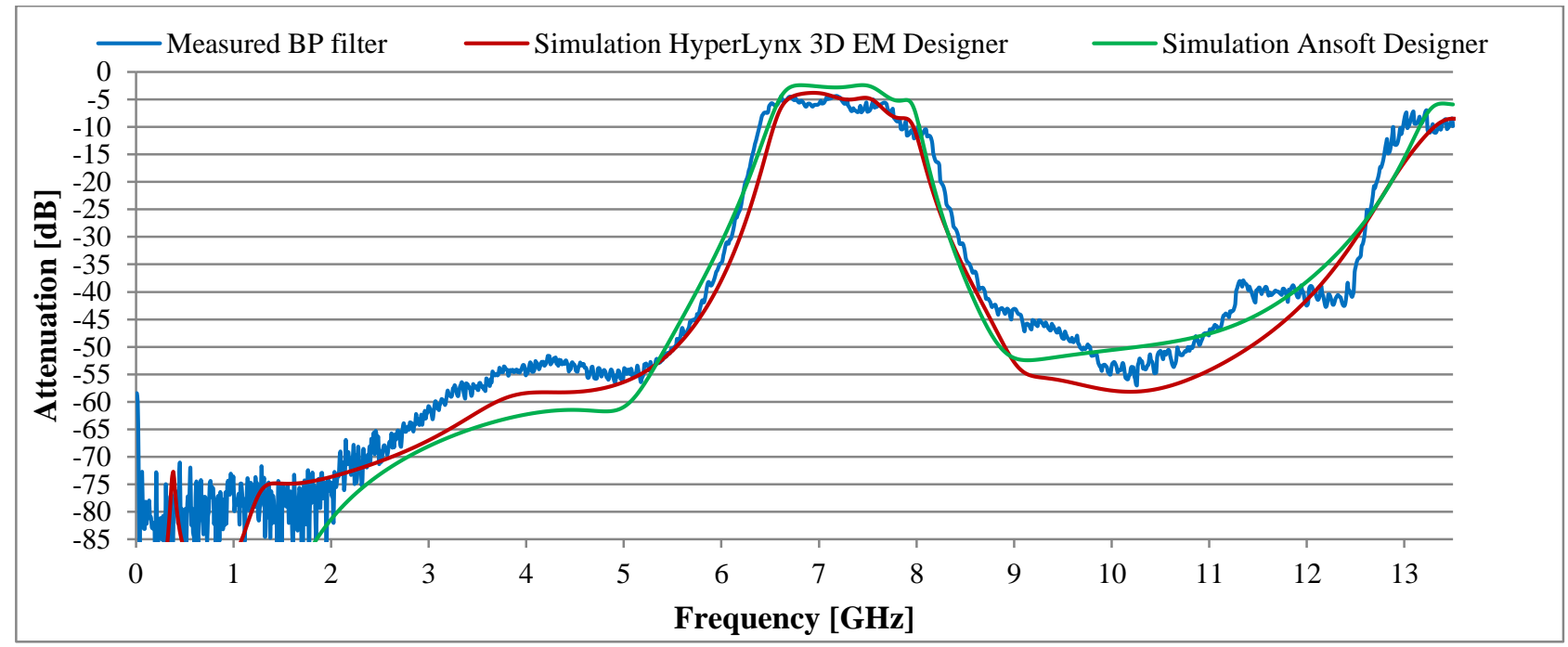

Fig. 7 Measured and simulated forward transmission coefficient of BP filter

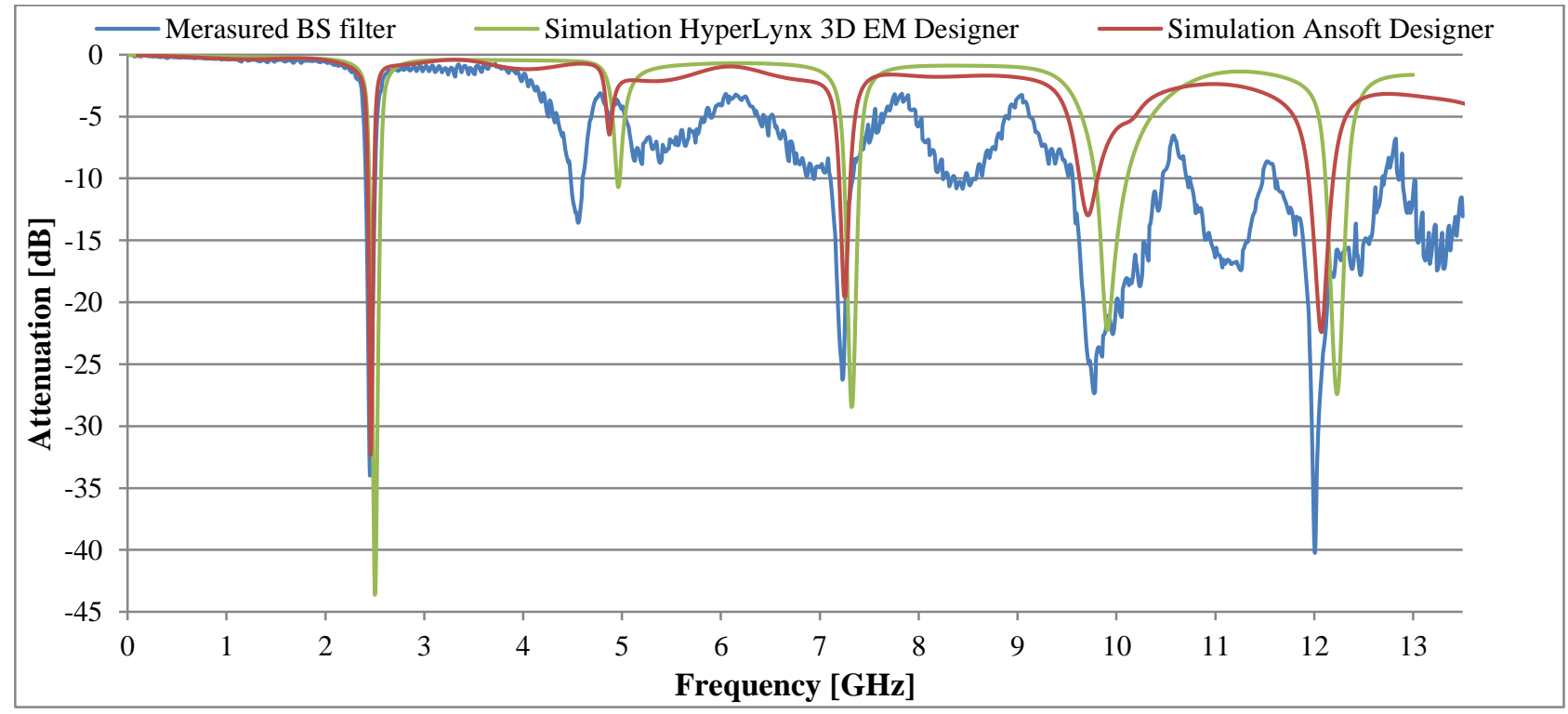

Fig. 8 Measured and simulated forward transmission coefficient of BS filter

\section{CONCLUSIONS}

Three types (LP, BP, BS) of filters were designed, simulated, fabricated and measured. Simulated and measured forward transmission coefficients were plotted in joint graphs to analyse their mutual correlation.

Simulations provided by Ansoft Designer are very ideal and do not correspond with real measurements, this distortion could influence performance of devices in very important manner, on the other hand, simulations provided by HyperLynx 3D EM Designer are in correlation with measured results, in general for all three types of filters. For these reasons HyperLynx 3D EM Designer is considered to be more suitable for design of microstrip structures in HF area.

\section{ACKNOWLEDGMENTS}

$*^{*^{* *}}$ The "We support research activities in Slovakia" project is co-financed from EU funds. This paper Európska únia was developed as part of the project named "Centre of Excellence for Integrated Research \& Exploitation of Advanced Materials and Technologies in Automotive Electronics", ITMS 26220120055.

\section{REFERENCES}

[1] ANSYS DesignerRF, Available on the Internet: 8.1.2014 http://www.ansys.com/Products/Simulation+Technol ogy/Electronics/RF+\&+Microwave. 
[2] HyperLynx 3D EM Designer, Available on the Internet:8.1.2015

http://www.scribd.com/doc/92415241/Ie3d-

Manual\#force_seo.

[3] ZHOU, J.: „Towards rational design of lowtemperature co-fired ceramic (LTCC) materials", Journal of Advanced Ceramics, 2012, pp. 89-99.

[4] Dupont Microcircuit Materials, Design and Layout Guidelines, Available on the Internet: 8.1.2015, http://www2.dupont.com/MCM/en_US/assets/downl oads/prodinfo/GreenTape_Design_Layout_Guideline s.pdf.

[5] DuPont LF171 Platinium Silver Conductor, Available on the Internet: 8.1.2015, http://www.dupont.com/content/dam/assets/products -and-services/electronic-electricalmaterials/assets/datasheets/prodlib/LF171.pdf.

[6] SACHS, J. - PEYERL, P. - KMEC, M. - TKAC, F.: „Digital Ultra-Wideband-Sensor Electronics integrated in SiGe-Technology", Proceedings of the EuMC, vol. 2, pp. 539-542, September 2002.

[7] JIA-SHENG HONG: "Microstip Filters for RF/Microwave Applications," John Wiley \& Sons, Inc., Hoboken, 2nd edition, New Jersey, 2011, 655.

[8] RUMAN, K. - PIETRIKOVÁ, A. - VEHEC, I. ROVENSKÝ, T. - GALAJDA, P.: „Integration of Microstrip LP and BP Filters to Multilayer Structure Based on Various LTCC" Electronics Technology (ISSE), 37th International Spring Seminar, Dresden, May 7-11, 2014 P. 114-119. ISBN 978-1-4799-44552.

[9] SACHS, J. - KMEC, M. - ZETIK, R. - PEYERL, P. - RAUSCHENBACH, P.: „Ultra wideband radar assembly kit", Geoscience and Remote Sensing Symposium, 2005, IGARSS ’05.

[10] ECC Decision (06)04, ,The harmonised conditions for devices using Ultra-Wideband (UWB) technology in bands below $10.6 \mathrm{GHz} " 2006$.

[11] RUMAN, K. - PIETRIKOVÁ, A. - VEHEC, I. GALAJDA, P.: „, Design of Microstrip Band Pass Filter Based on LTCC for UWB Sensor System "Electronics Technology (ISSE), 36th International Spring Seminar, Alba Iulia, May 8-12, 2013 P. $237-$ 241. ISBN 978-1-4799-0036-7.

[12] PIETRIKOVÁ, A. - ROVENSKÝ, T. - RUMAN, K - GAMEC, J.: „,Design of narrow-band $2.4-2.5 \mathrm{GHz}$ notch filter using various materials "Electronics
Technology (ISSE), 36th International Spring Seminar, Alba Iulia, May 8-12, 2013 P. 242-246. ISBN 978-1-4799-0036-7.

[13] ROVENSKÝ, T. - PIETRIKOVÁ, A. - RUMAN, K. - VEHEC, I.: „Stability of LTCC substrates in high frequency area after accelerated aging tests", Electronics Technology (ISSE), 37th International Spring Seminar, Dresden, May 7-11, 2014 P. 221224. ISBN 978-1-4799-4455-2.

Received January 16, 2015, accepted March 16, 2015

\section{BIOGRAPHIES}

Kornel Ruman was born on 16.12.1986. In 2011 he graduated (MSc) at the department of Electronics and Multimedia Communications of the Faculty of Electrical Engineering and Informatics at Technical University in Košice, his thesis title was "Projecting of FSO systems". Since 2011 started his stage at Department of Technologies in Electronics at Technical University in Košice as an PhD. Student. His dissertation thesis is "Contribution to the analysis of the behaviour of microstrip filters based on LTCC in UWB sensor systems". His research area is focused on the development of microstrip Low-pass and Band-pass filters for RF/Microwave Applications based on LTCC and on possibilities of use thick film technology in the high frequency area.

Tibor Rovenský was born on 1.4.1988. In 2012 he graduated (MSc) at the Department of Electronics and Multimedia Communications of the Faculty of Electrical Engineering and Informatics at Technical University in Košice. Since 2012 started his stage at Department of Technologies in Electronics at Technical University in Košice as a PhD. Student. His dissertation thesis is "Analysis of High Frequency Elements from the View of Different Materials and Technologies Applications'“. His research area is focused on the development of methods for dielectric properties measurements' and on analysing dielectric properties by this new developed method.

Alena Pietriková obtained M.Sc. in material science in 1980 and Ph.D. in 1986 at Technical University of Kosice, Slovakia. Currently she is professor of electro-technology and materials at the Department of Technologies in Electronics of the Faculty of Electrical Engineering and Informatics at Technical University in Kosice. She is coauthor of more than 230 scientific publications, 5 patents and 5 books. The most of her research works is focused on study of materials and technologies in electronics. 